\title{
RECONSTITUCIÓN DE TRÁNSITO ESOFÁGICO CON INTERPOSICIÓN DE COLON DERECHO*
}

\author{
Drs. Italo Braghetto M. ${ }^{1}$, Gonzálo Cardemil H. ${ }^{1}$, Attila Csendes J. ${ }^{1}$, \\ Carlos Domínguez C. ${ }^{1}$, Maher Musleh K. ${ }^{1}$, Owen Korn B. ${ }^{1}$, Hans Lembach J. ${ }^{1}$ \\ 1 Departamento de Cirugía, Hospital Clínico Universidad de Chile, Santiago. \\ Chile.
}

\begin{abstract}
Esophageal reconstruction using right colon interposition

Background: Esophageal replacement therapy is indicated for benign and malignant esophageal lesions and can be performed using stomach, jejunum or colon. Aim: To report the experience using right colon interposition for esophageal replacement. Material and Methods: Review of medical records of 41 patients aged 17 to 73 years ( 29 males), subjected to right colon interposition in the last 20 years. Indications, complications, mortality and long term results were recorded. Results: Forty nine percent of patients had a malignant esophageal lesion. Esophagectomy was performed using a transhiatal route or thoracotomy in 44 and $30 \%$ of patients, respectively. Retroesternal ascension was the most common route used in $88 \%$ of patients. The most common surgical complication was cervical fistula in $29 \%$ of patients, followed by fistula of the anastomosis between colon and jejunum in $7 \%$ of patients. Pneumonia was the most common medical complication. Postoperative mortality was $7 \%$. Conclusions: The mortality in this series of patients is similar to that reported elsewhere.
\end{abstract}

Key words: Esophageal reconstruction, right colon interposition, fistula.

\section{Resumen}

Introducción: La cirugía de reemplazo esofágico actualmente se indica para tratamiento de enfermedades benignas y malignas del esófago. Existen diversas técnicas de reconstitución de tránsito esofágico que utilizan estómago, yeyuno o colon. Objetivo: Presentar la experiencia acumulada en el Hospital Clínico de la Universidad de Chile utilizando la técnica de transposición de colon derecho para la reconstitución de tránsito esofágico. Materiales y Métodos: Estudio descriptivo restrospectivo que incluyó a los pacientes sometidos a reconstitución de tránsito esofágico mediante interposición de colon derecho, durante los últimos 20 años, basado en la revisión sistemática de historias clínicas, analizando causas, morbilidad precoz y tardía, mortalidad operatoria, compararándolos con los resultados de la literatura. Resultados: Se incluyen

\footnotetext{
*Recibido el 28 de octubre de 2014 y aceptado para publicación el 24 de noviembre de 2014.
}

Los autores no refieren conflictos de interés.

Correspondencia: Dr. Italo Braghetto M.

ibraghet@hcuch.cl 
41 pacientes operados, con edad promedio de 63,6 años (17-73), 29 de ellos de sexo masculino (70,7\%). El 48,7\% correspondieron a neoplasias malignas, el resto a patología benigna. En 43,9\% la esofaguectomía se realizó por vía transhiatal y $29,7 \%$ por toracotomía. El ascenso retrosternal fue la vía más empleada $(87,8 \%)$. La complicación más frecuente correspondió a la fístula cervical en $29,3 \%$ seguida de la fistula en colo-yeyuno anastomosis en el 7,3\% de los casos. La neumonía fue la complicación médica más frecuente $(14,7 \%)$. La mortalidad postoperatoria fue 7,3\%. Conclusión: El uso de interposición de colon derecho tiene indicaciones precisas. La mortalidad operatoria reportada se ajusta a lo encontrado en la literatura internacional.

Palabras clave: Esofaguectomía, tránsito esofágico, interposición de colon.

\section{Introducción}

La esofaguectomía es una intervención quirúrgica que produce un gran impacto psicológico y nutricional para los pacientes. Existen diversas técnicas de reconstitución de tránsito esofágico descritas, que utilizan tanto estómago, yeyuno o colon. El ascenso gástrico es considerado la primera alternativa para la reconstitución del tránsito esofágico. Sin embargo, existen situaciones en las cuales no es posible usar este órgano como sustituto del esófago ${ }^{1,2}$.

Presentamos experiencia acumulada en el Hospital Clínico de la Universidad de Chile utilizando la técnica de interposición de colon derecho en la reconstitución de tránsito esofágico, que ha sido la técnica de elección en nuestra unidad. Este trabajo se focaliza en el análisis de las indicaciones más frecuentes en este último período, las complicaciones intraoperatorias, las complicaciones postoperatorias precoces y tardías, su manejo y mortalidad postoperatoria.

\section{Materiales y Métodos}

Se diseñó un estudio descriptivo retrospectivo, basado en la revisión sistemática de historias clínicas de una serie de pacientes sometidos a reconstitución de tránsito esofágico mediante la técnica de interposición de colon derecho, efectuados durante los últimos 20 años en nuestro hospital. Se incluyen 41 pacientes con edad promedio 63,4 años, se excluyen otros tipos de interposición. Se registraron variables demográficas, clínicas y quirúrgicas. Se realizó análisis estadístico descriptivo utilizando medidas de tendencia central y dispersión, para lo cual se utilizó Excel 2011 y Stata 10SE. Se realiza revisión sistematizada de literatura de las publicaciones más recientes (PubMed) para comparar nuestros resultados; de 126 artículos se seleccionan 20 de ellos pues son los más atingentes al trabajo.

Rutinariamente se efectuó evaluación preoperatoria de colon para descartar enfermedad colónica inflamatoria o tumoral mediante colonoscopia y enema baritada.

\section{Técnica quirúrgica}

Después de la remoción del esófago, ya sea por vía toracotómica o toracoscópica, se cambia de posición al paciente en decúbito dorsal y se efectúa laparotomía media supraumbilcal lateralizada a derecha a nivel umbilical. Inicialmente se liberan completamente el colon derecho y transverso de sus mesos y epiplón mayor para inspeccionar la anatomía vascular de los segmentos colónicos determinando la distribución de los troncos arteriales y venosos a respetar para asegurar el adecuado suplemento sanguíneo al segmento a ascender. En nuestra experiencia se usó segmento de colon derecho e íleon terminal manteniendo el riego sanguíneo mediante los vasos cólicos derechos y medios, abriendo una ventana en el mesocolon, respetando los vasos cólicos medios y derechos y seccionando en su base el tronco de los vasos ileocecoapendicocólicos, manteniendo la anastomosis hacia el segmento de íleon distal (Figura 1). Se secciona el íleon terminal dejando un segmento de $20 \mathrm{~cm}$ desde la válvula ileocecal. El ascenso de colon en sentido isoperistáltico se hace por vía mediastínica o retroesternal y la anastomosis esófago-ileocolónica se efectúa a nivel cervical a través de cervicotomía vertical antero lateral izquierda usando puntos de material reabsorvible 000 a punto separado. Posteriormente se secciona el colon transverso respetando las arterias cólica media y derecha cerrando el muñón de colon tranverso con stapler o con sutura manual y luego se practica la anastomosis distal efectuándola a cara posterior de antro gástrico, duodeno o ángulo de Treitz según disponibilidad de estos segmentos. La íleo-coloanastomosis se efectúa latero-lateral a la tenia del muñón de colon tranverso ya sea con stapler o sutura manual absorvible en 2 planos. En todos los pacientes se efectúa control radiológico del tránsito digestivo con medio de contraste oral al $6^{\circ}$ día postoperatorio. Algunos pacientes tienen control endoscópico (Figuras 2 y 3 ). 

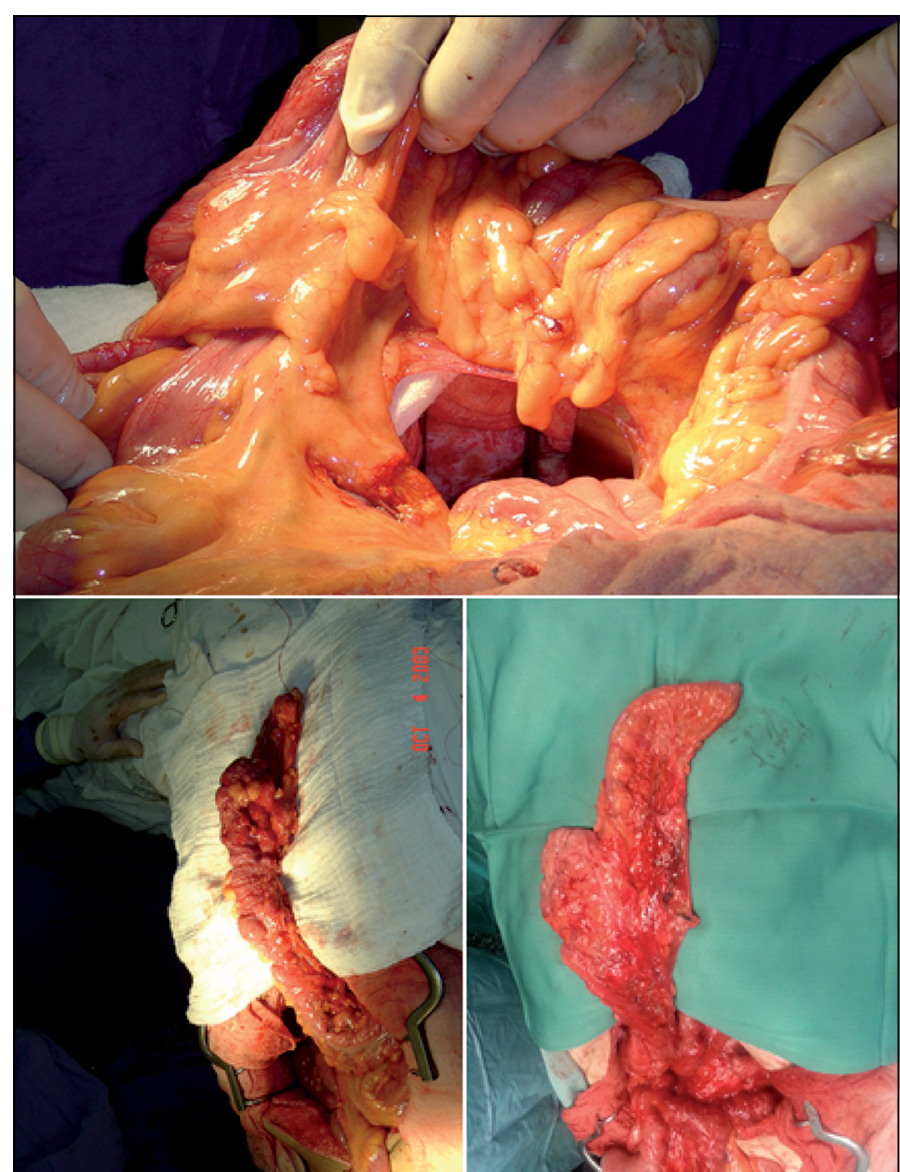

Figura 1. Preparación de colon derecho para su ascenso al cuello: se muestra la mantención de la irrigación del colon derecho y segmento de íleon distal.

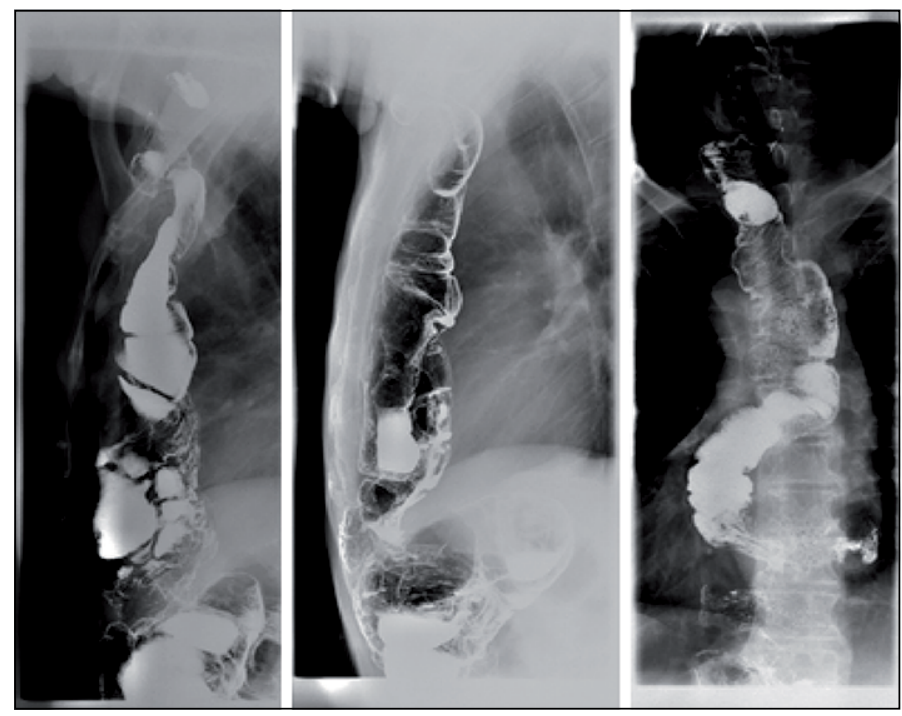

Figura 2. Control radiológico pos ascenso de colon derecho al cuello.

\section{Resultados}

De un total de 41 pacientes incluidos en este estudio, un $70,7 \%$ (29 pacientes) corresponde a sexo masculino, con una edad promedio de 63,6 años (53-78), sin diferencia entre sexos.

El $51,2 \%$ de los pacientes presentó una etiología de tipo benigna como causal de esófago-gastrectomía y el resto correspondió a etiologías malignas: cáncer esofágico $(26,8 \%)$, seguido por el cáncer de la unión gastroesofágica $(12,2 \%)$ y cáncer gástrico con infiltración hasta el esófago $(9,8 \%)$ (Tabla 1).

Las esofaguectomías fueron realizadas por vía transhiatal en 43,9\%, toracotomía derecha en $29,3 \%$ y en 1 paciente por toracoscopia. En 21,9\% se realizó un bypass esofágico, sin realizar esofaguectomía. La vía de ascenso de colon derecho al tórax fue realizada en $87,8 \%$ por vía retroesternal y en $12,2 \%$ por vía mediastínica. La mayor complicación intraoperatoria correspondió a una ruptura de la tráquea que obligó a su abordaje por toracotomía anterior, sutura y parche con pleura y grasa mediastínica, comprobándose en el intraoperatorio la ausencia de fuga aérea post procedimiento, la paciente evolucionó con una neumonía postoperatoria.

El 82,9\% presentó alguna complicación postoperatoria durante la hospitalización (Tablas 2 y 3). Las complicaciones quirúrgicas más frecuentes fueron la presencia de fístulas anastomóticas en $41,4 \%$, destacando la fístula de la esófago-íleo anastomosis presente en $29,3 \%$, fístula de la colo-yeyuno anastomosis en 3 pacientes (Tabla 2). Las complicaciones médicas más frecuentes fueron las complicaciones respiratorias en un 19,5\% (Tabla 3).

En estas Tablas se detalla el manejo de estas complicaciones, 14 pacientes debieron ser reoperados $(34,1 \%)$; dentro de los primeros 15 días del postoperatorio. Un paciente fue reoperado después de 1 año por estenosis de la anastomosis a nivel cervical sin respuesta a dilataciones endoscópicas.

La serie presenta una mortalidad global de $9,7 \%$, de los cuales $7,3 \%$ 


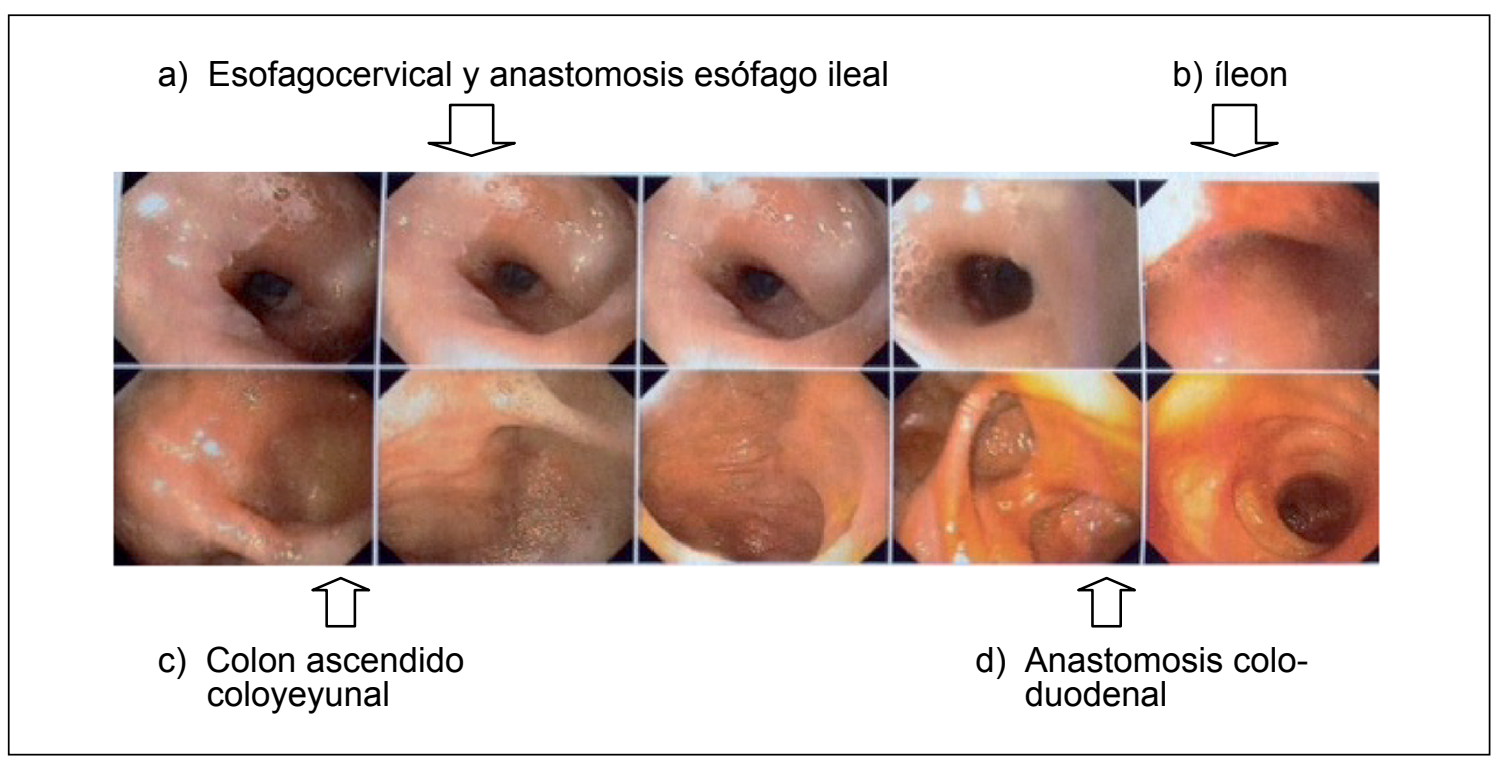

Figura 3. Control endoscópico post interposición de colon derecho.

Tabla 1. Etiología de enfermedades que indicaron realizar interposición de colon $(n=41)$

\begin{tabular}{|lrc|}
\hline Etiología & n & \% \\
Malignas & 20 & 48,8 \\
- Cáncer esófago & 11 & 26,8 \\
- Cáncer gástrico & 9 & 22,0 \\
Benignas & 21 & 51,2 \\
- Ingestión cáusticos & 10 & 24,4 \\
- Perforación esofágica & 7 & 17,0 \\
- Esofagitis severa & 4 & 9,8 \\
\hline
\end{tabular}

Tabla 2. Complicaciones postoperatorias del ascsenso de colon derecho $(n=41)$

\begin{tabular}{|lrcc|}
\hline Complicación & $\begin{array}{c}\mathbf{n} \\
\text { total }\end{array}$ & $\begin{array}{c}\mathbf{n} \\
\text { reoperados }\end{array}$ & $\begin{array}{c}\% \\
\text { reoperados }\end{array}$ \\
1. Filtración & & & \\
a. Esófago-colónica & 12 & 4 & 33 \\
$\begin{array}{l}\text { b. Colo-yeyunal } \\
\text { c. Íleo-colónica }\end{array}$ & 3 & 2 & 67 \\
d. Colo-duodenal & 1 & 1 & 100 \\
2. Hemoperitoneo & 2 & 2 & - \\
\hline 3. Obstrucción intestinal & 2 & 2 & 100 \\
4. Absceso subfrénico & 1 & 0 & - \\
\hline 5. Absceso cervical & 1 & 0 & - \\
6. Mediastinitis & 1 & 0 & - \\
\hline 7. Torácicos & 3 & 2 & 67 \\
\hline
\end{tabular}

corresponden a mortalidad postoperatoria y el resto, 1 paciente, atribuido a una complicación médica tardía.

En las Tablas 4 y 5 se muestran los datos reportados en la literatura en comparación con nuestros

Tabla 3. Complicaciones médicas del ascenso de colon derecho $(n=41)$

\begin{tabular}{|lccc|}
\hline Complicación & n & \% & \% reoperado \\
Neumonía & 5 & 12,2 & - \\
Derrame pleural & 2 & 4,8 & - \\
Empiema pleural & 1 & 2,4 & 100 \\
Insuficiencia respiratoria & 1 & 2,4 & - \\
\hline
\end{tabular}

Tabla 4. Indicaciones actuales para la interposición de colon para reemplazo del esófago: revisión de la literatura

\begin{tabular}{|lc|}
\hline Indicación & $\mathbf{\%}$ \\
\hline Cáncer de esófago o unión gastroesofágica & $37-61$ \\
\hline Imposibilidad de usar estómago & $38-84$ \\
\hline Estenosis benigna & $21-24$ \\
\hline Ingestión de cáusticos & $10-38$ \\
\hline Fístula esofágica iatrogénica & $2,4-8$ \\
\hline Reemplazo esofágico por causa benigna & $5-31$ \\
\hline Necrosis de sustituo esofágico previo & $3-10$ \\
\hline
\end{tabular}


Tabla 5. Complicaciones precoces y mortalidad postoperatoria: datos comparativos

\begin{tabular}{|lcc|}
\hline Complicaciones & $\begin{array}{c}\text { Literatura } \\
\%\end{array}$ & $\begin{array}{c}\text { U. de Chile } \\
\%\end{array}$ \\
\hline A. Médicas & $38-40$ & 22 \\
Respiratorias & 30 & 21 \\
\hline Tromboembolismo & $2-3$ & - \\
Sepsis & $6-8$ & 1 \\
\hline Cardiovasculares & $3-5$ & - \\
\hline B. Quirúrgicas & $33,3-60$ & 53 \\
\hline Fístula esofágica & $10-20$ & 29,7 \\
Fístula cologastro/coloyeyuno & $1,5-7$ & 7,3 \\
\hline Físula ileocolónica & $2-3$ & 2,4 \\
\hline Necrosis colon & $3-10$ & - \\
\hline Obstrucción intestinal & $5-8$ & 4,8 \\
\hline Quilotórax & $2-4$ & 2,4 \\
\hline Hemotórax & $2-18$ & 2,4 \\
\hline Hemorragia & $2-4$ & 2,4 \\
\hline Pancreatitis aguda & $2-3$ & - \\
\hline Reoperación & $4-38$ & 31 \\
\hline Mortalidad Postop & $2,5-17,5$ & $7,3 \%$ \\
\hline
\end{tabular}

resultados, en las Tablas 6 y 7 se muestran los síntomas y complicaciones alejadas reportadas, incluyendo nuestros resultados.

\section{Discusión}

En 1911 Vuillet y Kelling describieron el uso de colon como sustituto después de esofagectomía, técnica que no es muy frecuente ya que sólo en el 5\% se ha utilizado este órgano, sin embargo, este procedimiento se está usando cada vez más frecuentemente llegando en algunas series a ser utilizado en el $18 \%$ de las reconstituciones del tránsito digestivo ${ }^{3-10}$. Las indicaciones actuales incluyen causas malignas o causas benignas, por estenosis esofágica por cáusticos o péptica, fístula esofágica iatrogénica, mega esófago, necrosis del sustituto inicial del esófago (Tabla 4). En general, se emplea la interposición de colon debido a la imposibilidad de usar el estómago como resultado de una cirugía gástrica previa o resección gástrica sincrónica, daño gástrico por cáusticos o enfermedades previas o cirugía previa, por la necesidad de un conducto de mayor longitud que llegue hasta la faringe o por la posibilidad de un
Tabla 6. Síntomas alejados relacionados con complicaciones alejadas en interposición de colon. (Experiencia local y datos de literatura)

\begin{tabular}{|lc|}
\hline Disfagia & $\%$ \\
\hline Regurgitaciones & $0-50$ \\
\hline Sensación de plenitud & $2-20$ \\
\hline Pirosis & $10-16$ \\
\hline Síntomas respiratorios por aspiración & $8-14$ \\
Diarrea & $8-10$ \\
Baja de peso & $16-38$ \\
\hline
\end{tabular}

Tabla 7. Complicaciones tardías del ascenso de colon

\begin{tabular}{|lc|}
\hline & $\mathbf{\%}$ \\
\hline Estenosis cervical & $6-50$ \\
\hline Úlcera colónica & $3-5$ \\
\hline Obstrucción intestinal & $2-5$ \\
\hline Obstrucción retrosternal & $3-4$ \\
\hline Colon redundante & $2,8-10$ \\
\hline
\end{tabular}

largo tiempo de sobrevida post resección esofágica. En algunas series predominan las causas malignas, en otras en cambio son más frecuentes las causas benignas, dependiendo del centro hospitalario, región o del momento de la publicación ${ }^{3-8}$. Estas son las mismas causas que se observaron en nuestra casuística sólo que en las últimas décadas la ingestión de cáusticos con daño severo del esófago ha disminuido notablemente, actualmente la principal causa de esofaguectomía y consecuente indicación de su reemplazo con interposición de colon fue la cirugía de cáncer esofágico o de la unión gastroesofágica y sus complicaciones postoperatorias.

La evaluación preoperatoria del colon con enema baritada y colonoscopia es mandatoria para estudiar la longitud de los segmentos a utilizar y para descartar patología colónica. La arteriografía estaría indicada en pacientes añosos con sospecha de patología arterial crónica. Existen dos criterios esenciales a considerar para la elección del sustituto: la reconstrucción del esófago depende de la longitud requerida del injerto, y la anatomía vascular del colon $^{9,11}$. Para algunos el empleo de colon derecho con el segmento de íleon distal es una técnica fácil y segura, para otros cirujanos el uso del colon trans- 
verso con parte del colon ascendente es el sustituto de elección, posicionados en la dirección isoperistáltica. Para otros el segmento preferido es el colon izquierdo por la longitud que se puede obtener y por la menor variabilidad en la vascularización en contraste con el patrón vascular del lado derecho del colon $^{11,12}$. Autores japoneses utilizan con mayor frecuencia colon derecho con un segmento de íleon terminal para lograr segmentos más largos y con lúmenes similares al esófago con tránsito isoperistáltico ${ }^{13,14}$. En ausencia de estudios controlados, la elección entre una u otra opción finalmente se basa en la anatomía del suministro de sangre y de la preferencia y experiencia del cirujano. Al igual que los autores japoneses, en nuestra experiencia hemos preferido el uso de colon derecho isoperistáltico con un segmento de íleon terminal para ser anastomosado en el cuello con el esófago o faringe, dependiendo de la causa que originó la interposición de colon.

La ruta de ascenso es otro punto de controversia. La vía por mediastino posterior es más corta, más directa, evita la tensión, torsión o redundancia del segmento ascendido, puede acompañarse de mayores complicaciones pulmonares pero ofrece mejores resultados funcionales. En el estudio de Thomas ${ }^{11}$, la vía por el mediastino posterior se utilizó en el $63 \%$ de los casos. La coloplastía por vía retroesternal es el estándar de oro para la reconstrucción esofágica después de la lesión cáustica del tracto digestivo por el daño y secuelas en el mediastino posterior después de la perforación o necrosis esofágica por cáustico $^{5,7,9}$. El ascenso de colon puede tener complicaciones quirúrgicas como la dehiscencia de la anastomosis y sepsis debido a la necrosis de colon, por lo que muchos cirujanos usan una ruta retroesternal o subcutánea, ya que es más fácil de manejar la fuga anastomótica. A pesar de que esta técnica presenta mayor incidencia de fístula de la anstomosis a nivel cervical, es más manejable y no involucra mortalidad. Sin embargo, algunos investigadores han informado que la ruta del mediastino posterior ofrece mejores resultados funcionales a largo plazo. La isquemia no siempre es el resultado de la insuficiencia arterial, ya que también puede originarse por el deterioro del flujo sanguíneo venoso debido a una torsión del pedículo. Otros autores sugieren que el uso de colon izquierdo isoperistáltico por vía retrosternal $^{12,14}$. Para la reconstitucion del tránsito a nivel abdominal lo ideal es poder anastomosar el colon al remanente del estómago distal, de lo contrario será a nivel del duodeno o en el ángulo de Treitz ${ }^{8}$.

En manos expertas las tasas de mortalidad son inferiores al $5 \%$, pero las complicaciones postoperatorias (necrosis del injerto, fugas, estenosis de la anastomosis) son altos. La morbilidad global postoperatoria incluyendo complicaciones mayores o menores alcanza hasta un 45-64\%; dentro de las complicaciones más frecuentes están las fugas anastomóticas $(17,5 \%-20 \%)$. La necrosis de colon (5\%) es la complicación más temida, grave y fatal. La mortalidad varía entre un $2,5 \%$ a un $17,5 \% \%^{3,5,7-15}$. En general las complicaciones observadas en nuestra experiencia son muy similares, excepto la mayor incidencia de fístulas cervicales, que podría explicarse por la calidad intrínseca de nuestros pacientes y por el hecho de utilizar preferentemente un ascenso retrosternal, que presenta mayor riesgo de fístula; preferimos esta ruta por la benignidad y facilidad de manejo de una fístula cervical y sin riesgos de sepsis y mortalidad. La mortalidad operatoria reportada varía de un $2,5 \%$ a un $17,5 \%$, la nuestra fue de $7,3 \%$ (Tabla 5).

La reconstitución del tránsito con colon es la última oportunidad que tiene un paciente después de esofaguectomía y de la imposibilidad de otros sustitutos más simples (ascenso gástrico). Los resultados funcionales después de manejadas las complicaciones son bastante satisfactorios. Entre el $60-80 \%$ de los pacientes recuperan la posibilidad de alimentarse por vía oral y recuperan su condición nutricional después de la coloplastía. Disfunciones tardías post coloplastía (estenosis anastomóticas, redundancia de injerto) que requieren cirugía de revisión son frecuentes y podrían poner en peligro un resultado funcional (Tablas 6 y 7). La complicación alejada más frecuente es la disfagia, entre el 7,4\% y el $13 \%$ de los pacientes necesitan de dilataciones endoscópicas de la anastomosis esófago-ileal. Esta estenosis, de no ser tratada, puede llevar a neumonitis recurrentes por aspiración ${ }^{10-16}$.

Las complicaciones posteriores a la interposición de colon pueden ser agudas o crónicas, con frecuencia devastadoras ${ }^{17-19}$. Se necesitan estrategias creativas para solucionar esas complicaciones ${ }^{16-20}$. En un estudio de de Delva y cols..$^{17}$, las causas más frecuentes de reintervenciones son la redundancia del colon ascendido, la estenosis crítica de la anastomosis a nivel cervical y pérdida de la continuidad entre esófago cervical y el colon. Nosotros hemos comunicado nuestra experiencia de tratamiento alternativos cuando han ocurrido complicaciones post interposición de colon, lo que permite restituir la función de deglución en casi el $90 \%$ de lo casos $^{21}$.

Algunos autores sugieren el empleo de anastomosis vasculares micro-quirúgicas para aumentar el aporte sanguíneo al segmento ascendido con el objetivo de disminuir la isquemia, necrosis y fístulas postoperatorias $^{17-19}$; no tenemos experiencia con esta técnica. Autores japoneses han reportado disminución de la incidencia de fístula y $0 \%$ de pérdida del segmento colónico ascendido con una baja mortalidad operatoria ${ }^{17,20,21}$. 


\section{Referencias}

1. Postlethwait RW. Colonic interposition for esophageal substitution. Surg Gynecol Obstet. 1983;156:377-83.

2. Braghetto I, Csendes A, Amat J, Parada M, Schutte H, Lizana C, y cols. Reconstrucción del tránsito esofágico con interposición de colon. Rev Chil Cir. 1994;46:47784.

3. Thomas P, Fuentes P, Giudicelli R, Reboud E. Colon interposition for esophageal replacement: current indications and long-term function. Ann Thorac Surg. 1997;64:757-64.

4. Cseke L, Horváth OP. Indications, new surgical technique and results of colon interposition or bypass in esophageal surgery. Acta Chir Hung. 1997;36:49-50.

5. Cerfolio RJ, Allen MS, Deschamps C, Trastek VF, Pairolero PC. Esophageal replacement by colon interposition. Ann Thorac Surg. 1995;59:1382-4.

6. Wain JC, Wright CD, Kuo EY, Moncure AC, Wilkins EW Jr, Grillo HC, et al. Long-segment colon interposition for acquired esophageal disease. Ann Thorac Surg. 1999;67:313-7; discussion 317-8.

7. DeMeester TR, Johansson KE, Franze I, Eypasch E, Lu CT, McGill JE, et al. Indications, surgical technique, and long-term functional results of colon interposition or bypass. Ann Surg. 1988;208:460-74.

8. Boukerrouche A. Isoperistaltic left colic graft interposition via a retrosternal approach for esophageal reconstruction in patients with a caustic stricture: mortality, morbidity, and functional results. Surg Today 2014;44:827-33.

9. Monsour KA, Picone AZ, Coleman JJ. Surgery for high cervcal esophageal carcinoma: experience with 11 patients. Ann Thorac Surg. 1990:49:597-602.

10. Knezević JD, Radovanović NS, Simić AP, Kotarac MM, Skrobić OM, Konstantinović VD, et al. Colon interposition in the treatment of esophageal caustic strictures: 40 years of experience. Dis Esophagus 2007;20:530-4.

11. Thomas PA, Gilardoni A, Trousse D, D'Journo XB, Avaro JP, Doddoli C, et al. Colon interposition for oesophageal replacement. Multimed Man Cardiothorac Surg. 2009;(603):mmcts.2007.002956. doi: 10.1510/ mmcts.2007.002956.

12. Mine S, Udagawa H, Tsutsumi K, Kinoshita Y, Ueno M, Ehara K, et al. Colon interposition after esophagectomy with extended lymphadenectomy for esophageal cancer. Ann Thorac Surg. 2009;88:1647-53.

13. Matsumoto H, Hirai T, Kubota H, Murakami H, Higashida M, Hirabayashi Y. Safe esophageal reconstruction by ileocolic interposition. Dis Esophagus 2012;25:195-200.

14. Hamai Y, Hihara J, Emi M, Aoki Y, Okada M. Esophageal reconstruction using the terminal ileum and right colon in esophageal cancer surgery. Surg Today 2012;42:342-50.

15. Chirica $M$, de Chaisemartin C, Muñoz-Bongrand $N$, Halimi B, Celerier M, Cattan P, et al. Colonic interposition for esophageal replacement after caustic ingestion. J Chir. (París) 2009;146:240-9.

16. Oida T, Mimatsu K, Kano H, Kawasaki A, Kuboi Y, Fukino N, et al. Anterior vs posterior mediastinal routes in colon interposition after esophagectomy. Hepatogastroenterology 2012;59:1832-4.

17. de Delva PE, Morse CR, Austen WG Jr, Gaissert HA, Lanuti M, Wain JC, et al. Surgical management of failed colon interposition. Eur J Cardiothorac Surg. 2008;34:432-7.

18. Kesler KA, Pillai ST, Birdas TJ, Rieger KM, Okereke IC, Ceppa D, et al. "Supercharged" isoperistaltic colon interposition for long-segment esophageal reconstruction. Ann Thorac Surg. 2013;95:1162-8; discussion 1168-9.

19. Blackmon SH, Correa AM, Skoracki R, Chevray PM, Kim MP, Mehran RJ, et al. Supercharged pedicled jejunal interposition for esophageal replacement: a 10-year experience.Ann Thorac Surg. 2012;1104-11; discussion 1111-3.

20. Shirakawa Y, Naomoto Y, Noma K, Sakurama K, Nishikawa T, Nobuhisa T, et al. Colonic interposition and supercharge for esophageal reconstruction. Langenbecks Arch Surg. 2006;391:19-23.

21. Braghetto I, Cardemil G, Csendes A, Venturelli A, Herrera M, Korn O, et al. Digestive tract reconstitution after failed esophago-gastro or esophago-coloanastomosis. Arq Bras Cir Dig. 2013;26:7-12. 